\title{
Global immune fingerprinting in glioblastoma patient peripheral blood reveals immune-suppression signatures associated with prognosis
}

Tyler J. Alban, ${ }^{1,2}$ Alvaro G. Alvarado, ${ }^{3}$ Mia D. Sorensen, ${ }^{4,5}$ Defne Bayik, ${ }^{1}$ Josephine Volovetz, ${ }^{1,2}$ Emily Serbinowski, ${ }^{1}$ Erin E. Mulkearns-Hubert, ${ }^{1}$ Maksim Sinyuk, ${ }^{1}$ James S. Hale, ${ }^{1}$ Giovana R. Onzi, ${ }^{3,6}$ Mary McGraw, ${ }^{7}$ Pengjing Huang,' Matthew M. Grabowski, ${ }^{7,8}$ Connor A. Wathen, ${ }^{2,7}$ Manmeet S. Ahluwalia, ${ }^{7,9}$ Tomas Radivoyevitch, ${ }^{10}$ Harley I. Kornblum, ${ }^{3}$ Bjarne W. Kristensen, ${ }^{4,5}$ Michael A. Vogelbaum, ${ }^{2,7,8,9}$ and Justin D. Lathia ${ }^{1,2,7,9}$

'Department of Cellular and Molecular Medicine, Lerner Research Institute, Cleveland Clinic, Cleveland, Ohio, USA. ${ }^{2}$ Department of Molecular Medicine, Cleveland Clinic Lerner College of Medicine of Case, Western Reserve University, Cleveland, Ohio, USA. ${ }^{3}$ Department of Psychiatry and Biobehavioral Sciences and Semel Institute for Neuroscience, UCLA, USA. ${ }^{4}$ Department of Pathology, Odense University Hospital, Odense, Denmark. ${ }^{5}$ Department of Clinical Research, University of Southern Denmark, Odense, Denmark. ${ }^{6}$ Department of Biophysics and Center of Biotechnology, Federal University of Rio Grande do Sul (UFRGS), Porto Alegre, RS-Brazil. 'Rose Ella Burkhardt Brain Tumor and Neuro-Oncology Center and ${ }^{8}$ Department of Neurosurgery, Cleveland Clinic, Cleveland, Ohio, USA. ${ }^{9}$ Comprehensive Cancer Center, Case Western Reserve University, Cleveland, Ohio, USA. ${ }^{10}$ Department of Quantitative Health Sciences, Lerner Research Institute, Cleveland Clinic, Cleveland, Ohio, USA.

Clioblastoma (CBM) remains uniformly lethal, and despite a large accumulation of immune cells in the microenvironment, there is limited antitumor immune response. To overcome these challenges, a comprehensive understanding of CBM systemic immune response during disease progression is required. Here, we integrated multiparameter flow cytometry and mass cytometry TOF (CYTOF) analysis of patient blood to determine changes in the immune system among tumor types and over disease progression. Utilizing flow cytometry analysis in a cohort of $\mathbf{2 5 9}$ patients ranging from benign to malignant primary and metastatic brain tumors, we found that CBM patients had a significant elevation in myeloid-derived suppressor cells (MDSCs) in peripheral blood but not immunosuppressive Tregs. In CBM patient tissue, we found that increased MDSC levels in recurrent CBM portended poor prognosis. CyTOF analysis of peripheral blood from newly diagnosed GBM patients revealed that reduced MDSCs over time were accompanied by a concomitant increase in DCs. CBM patients with extended survival also had reduced MDSCs, similar to the levels of lowgrade glioma (LCG) patients. Our findings provide a rationale for developing strategies to target MDSCs, which are elevated in GBM patients and predict poor prognosis.

Authorship note: MAV and JDL are co-senior authors.

Conflict of interest: The authors have declared that no conflict of interest exists.

License: Copyright 2018, American Society for Clinical Investigation.

Submitted: May 16, 2018

Accepted: October 2, 2018

Published: November 2, 2018

Reference information: JCI Insight. 2018;3(21):e122264. https://doi.org/10.1172/jici. insight.122264.

\section{Introduction}

Glioblastoma (GBM) is the most prevalent primary malignant brain tumor and remains uniformly fatal despite aggressive therapies, including surgery, radiation, and chemotherapy (1). Currently, there is great interest in targeting the immune system to promote antitumor response as a new means of treating cancers, including GBM (2-4). Despite the presence of potential antitumor effector cells within the microenvironment, GBM growth persists (5-14). One possible explanation for the lack of effective antitumor immune response is the presence of an immunosuppressive microenvironment $(7,12-14)$. This is likely due to a number of factors, including immune checkpoint signaling, $\mathrm{T}$ cell exhaustion, glucose depletion, hypoxia, and the presence of immunosuppressive cells, such as Tregs, tolerogenic DCs, and myeloid-derived suppressor cells (MDSCs) (12, $14,15)$. Another factor that could contribute to the limited immune response is the low mutational load of GBM, which does not allow for the recognition and removal of cancer cells by the immune system (16). All of these factors combined have led to the testing of checkpoint inhibitors in clinical trials, which demonstrated 
that the antigen-specific $\mathrm{T}$ cell responses do not always correlate with tumor regression, suggesting that the immunosuppressive microenvironment limits the potential of $\mathrm{T}$ cell activation $(17,18)$. While this degree of immunosuppression in GBM appears extreme (19), it is consistent with the unique immunosuppressive architecture off the brain and may, thus, be more difficult to reverse than with tumors in other locations $(4,20,21)$.

Given these barriers to the use of immunotherapy approaches, identifying mechanisms of peripheral and tumoral immunosuppression in GBM is an immediate priority. Here, we aimed to determine whether GBM patients have an elevation of circulating peripheral immunosuppressive cells compared with other brain tumor patients, determine if intratumoral MDSCs are associated with prognosis, and identify the overall immune cell profile in the peripheral blood of GBM patients over time. MDSCs are of particular interest given their previously identified role in GBM immunosuppression, immunotherapy response, and cancer progression (12, $13,15,22,23)$. A number of contact-dependent and contact-independent pathways have been described for MDSCs, which broadly inhibit T cell proliferation and activation (24). MDSC production is induced following an inflammatory response to restore homeostasis (25). However, it has been demonstrated that MDSCs are also increased in most, if not all, cancers in which they have been examined (26). In addition, it is not surprising that MDSCs are also increased in GBM, considering the consequences of large-scale inflammation in the brain (27). This opens up the possibility that, while MDSCs could be induced by cancer cells to help evade immune recognition, they may also be recruited by healthy brain cells such as microglia and astrocytes to protect the brain from excessive inflammation (28). We recently identified an interaction between MDSCs and GBM cancer stem cells via macrophage migration inhibitory factor (MIF), leading to enhanced MDSC function and increased cytotoxic T cell infiltration, which could be targeted to reduce GBM growth (22). However, due to the intricate and interconnected nature of the immune system, it is not clear how targeting a single immunosuppressive cell pathway would impact the function of the antitumoral immune system. Taken together, this suggests that there is a need to delineate the complex nature of the GBM immune response.

The immune alterations in GBM have primarily been examined using targeted approaches such as immunohistochemical staining and flow cytometry and unbiased approaches such as RNA sequencing (RNA-seq) analysis (16, 29, 30). GBM immunohistochemical analysis has been useful for identifying infiltrating macrophages/monocytes, Tregs, and T cell dysfunction $(6,14,31)$. While immunohistochemical and immunofluorescent staining techniques are becoming more advanced, they fail to provide a general picture of the immune system within GBM based on their marker number limitations (32). Flow cytometry has similar pitfalls; although flow cytometry has been used successfully to identify immune cell infiltration and dysfunction in GBM, it is limited by the number of fluorescent markers that can be used due to compensation issues and overlap in fluorophore signals (33). Finally, RNA-seq studies have been able to profile the immune response in GBM compared with other cancers, but this is a recent development in which the relative abundance of immune cell populations is determined using TCGA pan-cancer data (16). These RNA-seq studies have determined that intratumoral MDSCs, Tregs, and effector memory CD4 ${ }^{+} \mathrm{T}$ cells are the most prevalent immune cell populations in GBM, with MDSCs enriched in more than $70 \%$ of patients with a low mutational burden (16). While RNA-seq provides new insights into the immune landscape of GBM, these analyses have been performed on bulk tumors and are, thus, not an ideal way to examine multiple immune cell lineages to determine the composition of the immune microenvironment. An emerging technology with the potential to reveal the composition of the immune microenvironment of many cancers is mass cytometry TOF (CyTOF) (34-36). CyTOF can identify immune cell response and differentiation by simultaneously assessing a high number of markers, which cannot be performed by traditional techniques (30). This approach is currently being used in multiple cancers to examine the immune landscape of tumors to identify how to best enhance the antitumor immune response $(30,34,35)$. Here, we use a combination of approaches, including flow cytometry, immunofluorescence, and CyTOF, to identify an immunosuppressive phenotype with increased MDSCs and reduced antitumoral response in GBM patients with a poor prognosis compared with low-grade glioma (LGG) patients and GBM patients with a good prognosis.

\section{Results}

Peripheral blood MDSCs are increased in GBM patients compared with other brain tumor patients, and intratumoral MDSCs are predictive of patient prognosis. To quantify immunosuppressive MDSCs in brain tumor patients, peripheral blood mononuclear cells (PBMCs) were isolated from patients undergoing surgical resection (Figure 1A). Samples were analyzed via flow cytometry using an MDSC-focused panel of antibodies against CD11b, HLA-DR, CD14, CD15, and CD33 (Supplemental Figure 1, A and C; supplemental material available online 
A

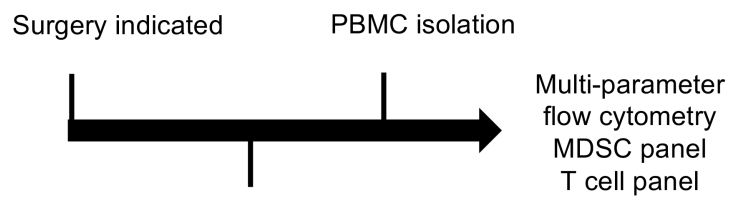

Intraoperative blood draw
B

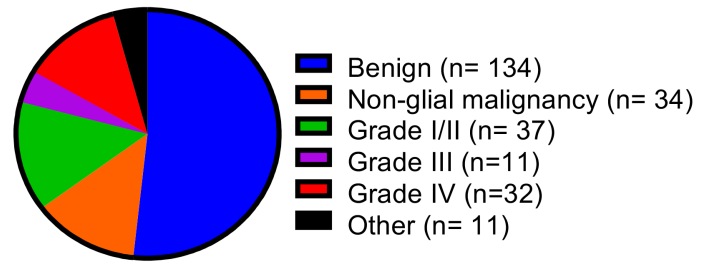

D T Regulatory Cells

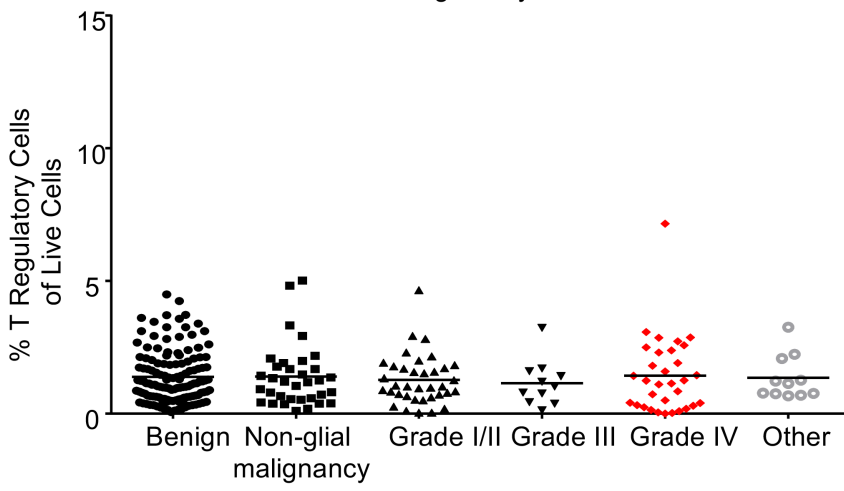

$\mathbf{F}$
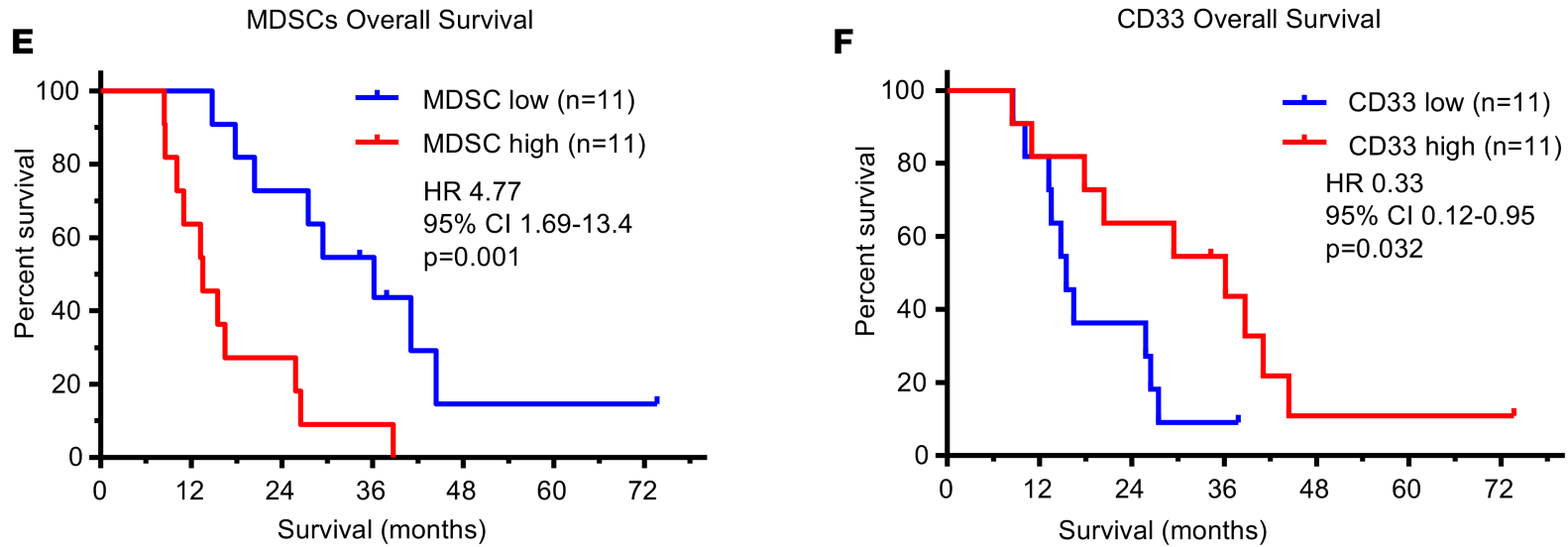

Figure 1. Patient analysis identifies peripheral and tumoral MDSCs associated with glioma grade and patient prognosis. (A) Experimental design: patients entering the clinic for surgical resection were consented, and a blood sample was acquired intraoperatively. Subsequently, PBMCs were isolated via Ficoll-Paque gradient within 24 hours before being frozen in freezing media for future use. (B) Pie chart with the distribution of patient samples totaling $n=259$ patients analyzed. (C and $\mathbf{D})$ Analysis of immunosuppressive M-MDSCs and Tregs via multiparameter flow cytometry analysis, where individual unpaired 2-tailed Student's $t$ tests were used and then corrected with Benjamini-Hochberg method (horizontal lines represent mean values, $\left.{ }^{* *} P<0.01,{ }^{* *} P<0.001\right)$. (E) Kaplan-Meier analysis of patients separated by median levels of MDSC signal in the CD33 ${ }^{+}$area demonstrates decreased survival $(P=0.001)$. Statistical significance evaluated by log-rank analysis $(n=22)$. (F) Kaplan-Meier analysis of patients divided by median CD33 levels identifies increased overall survival using log-rank test $(P=0.032, n=22)$.

with this article; https://doi.org/10.1172/jci.insight.122264DS1). For comparison, a separate $\mathrm{T}$ cell-focused panel containing antibodies against $\mathrm{CD} 3, \mathrm{CD} 4, \mathrm{CD} 25, \mathrm{CD} 8, \mathrm{CD} 107 \mathrm{a}$, and $\mathrm{CD} 127$ was used to quantify cytotoxic $\mathrm{T}$ cells and Tregs (Supplemental Figure 1, B, D, and E). The patient cohort for these studies consisted of 259 patients, who were subdivided into the categories benign, nonglial malignancy, and glial malignancy (grade I/II, grade III, grade IV [GBM]) (Figure 1B, with further final diagnoses for each group elaborated on in Supplemental Figure 2). When MDSC levels, as determined by the percent of HLA-DR ${ }^{-10} / \mathrm{CD} 33^{+} / \mathrm{CD}_{11 b^{+}}$ cells of the total live cells, were compared across groups, we observed that benign samples had a lower percentage of MDSCs compared with nonglial malignancies and grade IV glioma samples — but not grade I/II or III glioma samples (Figure 1C). Additionally, nonglial malignancies had increased MDSCs compared with grade I/II tumors but not grade III or IV glioma, suggesting that MDSCs may be a possible marker of malignancy in brain tumor patients (Figure 1C). A direct comparison among glial malignancies within the categories of grade I/II, -III, and -IV revealed that grade I/II tumors had significantly lower numbers of MDSCs compared with 
Table 1. Univariable analysis identifies MDSCs as a predictor of WHO glioma grade

\begin{tabular}{lc}
\hline & Univariable linear model analyses of MDSC \\
Variable & $P$ value \\
Chronic steroid use & 0.063 \\
Ki67 index & 0.660 \\
IDH1 status & 0.875 \\
WHO grade & 0.016 \\
Age & 0.045 \\
Sex & 0.185 \\
MGMT status & 0.794 \\
Univariable modeling of MDSC association with tumor diagnosis ( $n=259)$. Univariable linear model fits show that \\
only grade associates with M-MDSCs $(P \leq 0.05)$ MGMT, methylguanine-DNA methyltransferase; IDH1, isocitrate \\
dehydrogenase; MDSC, myeloid-derived suppressor cell.
\end{tabular}

grade IV samples, confirming results found by others (Figure 1C) (15). Univariate analysis of dependence of MDSC levels on age, sex, grade, isocitrate dehydrogenase 1 (IDH1) mutation status, $\mathrm{O}^{6}$-methylguanine-DNA methyltransferase (MGMT) status, and chronic steroid use prior to surgery (Table 1) yielded World Health Organization (WHO) grade as the most significant predictor of MDSC levels $(P=0.016)$. This remained significant in multivariable models that controlled for the potentially confounding clinical variables of age $(P=$ $0.016-0.076)$ and chronic steroid use $(P=0.016-0.053)$ (i.e., other variables marginally significant if modeled alone). To determine whether other immunosuppressive cell types in circulation are also increased with malignancy, we assessed Tregs as the percentage of $\mathrm{CD}^{+} / \mathrm{CD}^{+} / \mathrm{CD} 8^{-} / \mathrm{CD} 127^{-} / \mathrm{CD} 25^{+}$cells (Figure $1 \mathrm{D}$ and Supplemental Figure 1E). No statistically significant differences were observed for Tregs among the categories of benign tumors, nonglial malignancies, or glial malignancies. These results demonstrate a relationship between circulating MDSCs and tumor grade, but not between Tregs and tumor grade.

Immunofluorescence staining of matched primary and recurrent GBM tumors identifies a correlation between $M-M D S C$ s and survival. To validate our observation that circulating MDSCs were associated with increased malignancy, we utilized immunofluorescence analysis of MDSCs in matched primary and recurrent tumor samples from 22 GBM patients via antibody staining for CD33, IBA1, and HLA-DR (Supplemental Figure 3). IBA1 was used in place of CD11b because CD11b marks both neutrophils (CD11b ${ }^{+}, \mathrm{IBA1}^{-}$, $\left.\mathrm{CD}^{+}, \mathrm{HLA}^{+} \mathrm{DR}^{-/+}\right)$and granulocytic MDSCs $\left(\mathrm{CD} 11 \mathrm{~b}^{+}, \mathrm{IBA}^{-}, \mathrm{CD}^{+}, \mathrm{HLA}^{+} \mathrm{DR}^{-/+}\right)$, while IBA1 marks monocytic MDSCs (M-MDSCs) (IBA1 ${ }^{+}, \mathrm{CD}_{3}{ }^{+}, \mathrm{HLA}_{-\mathrm{DR}}{ }^{-}$) and microglia (IBA1 ${ }^{+}, \mathrm{CD} 33^{\mathrm{lo}}, \mathrm{HLA}-\mathrm{DR}{ }^{+}$), which are easily distinguished by CD33 and HLA-DR levels (37-39). Within this cohort, patients were treated with a similar clinical paradigm (radiation and concomitant chemotherapy via the Stupp protocol; ref. 1). Patients with high and low MDSC levels were identified by the median area of MDSCs (HLA$\mathrm{DR}^{1 \mathrm{o} /-} / \mathrm{IBA}^{+} / \mathrm{CD}^{2} 3^{+}$) relative to total tumor area (Supplemental Figures 3 and 4 ; Supplemental Table 1 and 2). To determine whether MDSC levels were associated with patient outcome, patients were divided into $\mathrm{MDSC}^{\text {hi }}$ and $\mathrm{MDSC}^{\text {lo }}$ groups based on their median level of MDSCs at primary and recurrent resections, where overall survival, time between first and second surgery, survival after second surgery, and progression-free survival were analyzed (Table 2). In this analysis, we found that grouping patients based on primary levels of MDSCs was not predictive of patient outcome, while grouping on recurrent levels of MDSCs was predictive of survival (Table 2). Overall, these analyses indicated that MDSC levels at primary resection were not predictive of prognosis but that MDSC levels during recurrence were informative for overall survival, time between first and second surgery, and survival after second surgery (Figure 1, E and F, and Supplemental Figures 4 and 5). Based on the observation that MDSC ${ }^{\text {hi }}$ patients had a significantly reduced overall survival compared with $\mathrm{MDSC}^{\text {lo }}$ patients (Figure 1E), we sought to determine whether this was specific to MDSCs or whether myeloid cell levels $\left(\mathrm{CD} 33^{+}\right)$were also predictive of survival. This was not the case for overall myeloid cells, as assessed by CD33 expression, as high myeloid cell numbers were associated with increased survival (Figure $1 \mathrm{~F}$ and Supplemental Figure 4, A and B). A Cox regression analysis yielded MDSCs and CD33 levels as the most predictive variables (Supplemental Tables 1 and 2). These findings demonstrate that high levels of MDSCs upon recurrence portends poor prognosis, while infiltration of other subtypes of myeloid cells is beneficial. 
Table 2. Log rank analysis of MDSC levels in primary and recurrent GBM specimens reveals that recurrent MDSCs are predictive of prognosis

\begin{tabular}{|c|c|c|c|c|c|c|c|c|c|}
\hline & & & & & & $\begin{array}{l}\text { Overall } \\
\text { survival }\end{array}$ & $\begin{array}{c}\text { Time between } \\
\text { first and } \\
\text { second surgery }\end{array}$ & $\begin{array}{c}\text { Survival } \\
\text { after second } \\
\text { surgery }\end{array}$ & $\begin{array}{l}\text { Progression- } \\
\text { free survival }\end{array}$ \\
\hline \multirow{9}{*}{ Primary } & \multirow{9}{*}{$\mathrm{CD} 3^{+}$} & \multirow{9}{*}{ IBA $1^{+}$} & \multirow{3}{*}{ HLA-DR- } & \multirow{2}{*}{ Total tumor area } & Spearman $r$ & 0.18 & \begin{tabular}{l|}
0.16 \\
\end{tabular} & -0.018 & 0.28 \\
\hline & & & & & Log Rank ( $P$ value) & 0.18 & 0.21 & 0.36 & 0.12 \\
\hline & & & & $\mathrm{CD}_{3} 3^{+}$area & Spearman $r$ & 0.075 & 0.13 & -0.16 & 0.25 \\
\hline & & & \multirow{6}{*}{ HLA-DR ${ }^{10}$} & \multirow{3}{*}{ Total tumor area } & Spearman $r$ & 0.090 & 0.027 & 0.022 & 0.12 \\
\hline & & & & & Spearman $P$ value & 0.69 & 0.91 & 0.92 & 0.59 \\
\hline & & & & & Log Rank ( $P$ value) & 0.22 & 0.28 & 0.37 & 0.16 \\
\hline & & & & \multirow{3}{*}{$\mathrm{CD} 3^{+}$area } & Spearman $r$ & 0.089 & 0.12 & -0.20 & 0.21 \\
\hline & & & & & Spearman $P$ value & 0.69 & 0.58 & 0.36 & 0.35 \\
\hline & & & & & Log rank ( $P$ value) & 0.78 & 0.39 & 0.66 & 0.25 \\
\hline \multirow{9}{*}{ Recurrent } & \multirow{9}{*}{$\mathrm{CD}^{+} 3^{+}$} & \multirow{9}{*}{$\mathrm{IBA}^{+}$} & \multirow{3}{*}{ HLA-DR- } & \multirow{3}{*}{$\mathrm{CD} 3^{+}$area } & Spearman $r$ & -0.61 & -0.48 & -0.67 & -0.35 \\
\hline & & & & & Spearman $P$ value & 0.003 & 0.024 & 0.001 & 0.11 \\
\hline & & & & & Log rank ( $P$ value) & 0.0013 & 0.14 & 0.0006 & 0.22 \\
\hline & & & \multirow{6}{*}{ HLA-DR ${ }^{10}$} & \multirow{3}{*}{ Total tumor area } & Spearman $r$ & 0.002 & -0.13 & -0.086 & -0.031 \\
\hline & & & & & Spearman $P$ value & 0.99 & 0.56 & 0.70 & 0.89 \\
\hline & & & & & Log rank ( $P$ value) & 0.30 & 0.53 & 0.31 & 0.40 \\
\hline & & & & \multirow{3}{*}{$\mathrm{CD} 3^{+}$area } & Spearman $r$ & -0.27 & -0.28 & -0.39 & -0.18 \\
\hline & & & & & Spearman $P$ value & 0.22 & 0.20 & 0.073 & 0.42 \\
\hline & & & & & Log rank ( $P$ value) & 0.74 & 0.94 & 0.76 & 0.89 \\
\hline
\end{tabular}

Log-rank analysis of MDSCs separated by HLA-DR and HLA-DR ${ }^{10}$ populations from primary or recurrent levels, where correlation with survival, time between surgeries, survival after second surgery, and progression-free survival were analyzed $(n=22)$. Spearman rank correlation coefficients are also provided as indicators of association magnitude and directionality.

Longitudinal study of GBM patients using an immune-fingerprinting approach via CyTOF reveals changes over disease progression. To determine how MDSCs and the overall immune system of GBM patients change during disease progression, samples from a cohort of 6 newly diagnosed GBM patients were analyzed via multiparameter flow cytometry and CyTOF (Supplemental Figure 6). Blood draws from these patients were obtained during surgery, 2 weeks after surgery, and then every 2 months until the patient left the study or succumbed to disease (Figure 2A). CyTOF with a panel of 25 immune cell markers (Supplemental Figure 7 and 8) was used for an in-depth analysis of how the immune system is altered during disease progression. Unbiased clustering was performed to determine whether differences existed between the baseline values of patients and subsequent time points, and we observed that baseline samples grouped to one side of a multidimensional scaffolding (MDS) plot, indicating differences between the baseline and subsequent time points (Figure 2B) (40). To identify cell type-specific clusters, a t-distributed stochastic neighbor embedding (tSNE) analysis was performed and, in an unbiased manner, identified 30 unique clusters of cells by taking into account 25 immune markers. The 30 clusters were then grouped into 12 immune cell types based on the histogram of marker expression within each cluster (Figure 2C and Supplemental Figure 9). Performing the same cluster analysis on each sample individually allowed visualization of how each immune cell cluster changed over time relative to the entire immune profile (Supplemental Figure 10). Because it has been observed that GBM patients with IDH1 mutations have altered tumor progression and immune populations (41-44), we started our study by performing a direct comparison of patient 2 (IDH1 mutant) and patient 4 (IDH1 WT), because of their differences in MDSCs (and thus prognosis), identified by flow cytometry (Supplemental Figure 6 and 11). For this comparison, we performed FlowSOM analysis - as well as manual gating for MDSCs, NK cells, and DCs - and found that patient 2 (IDH1 mutant) had low MDSCs with high numbers of antitumoral 
A

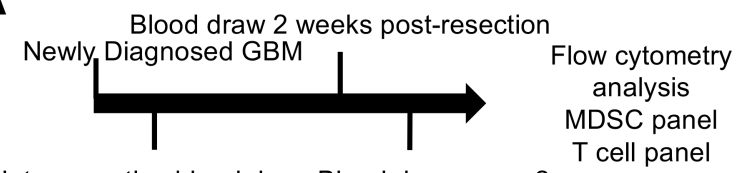

Intraoperative blood draw Blood draw every 2 CyTOF panel months post- follow up

B

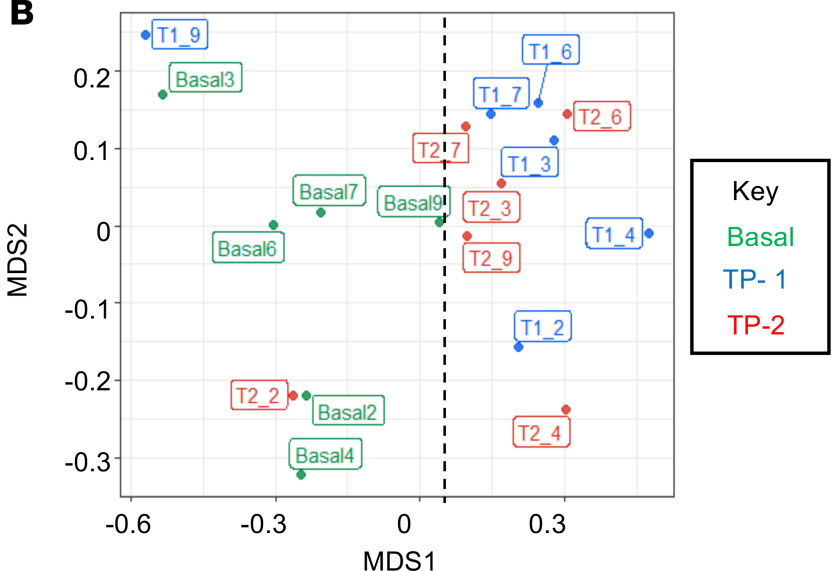

C

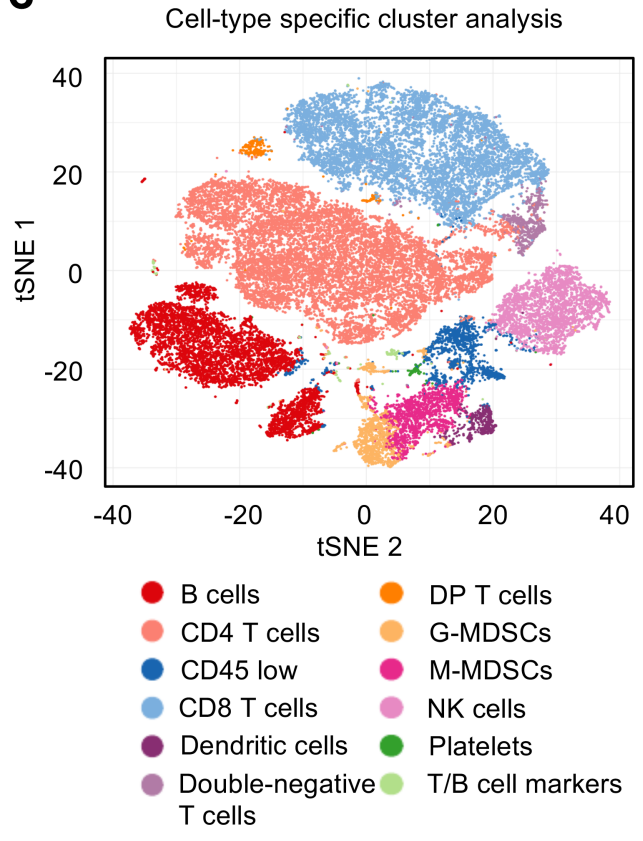

Figure 2. Mass cytometry analysis of GBM patients over time reveals immune shifts from baseline that are not common across all patients. (A) Schematic representation of the patient cohort consisting of $n=6$ glioblastoma patients followed over time, with blood collection and storage for analysis via multiparameter flow cytometry and CyTOF. (B) Multidimensional scaffold plot representing 6 patients at 3 time points each (baseline, time point 1, and time point 2). The first number represents the time point and the second represents the patient. Dotted line represents the division between baseline samples and later time point samples. (C) tSNE plot identifies 30 unique populations that are color coded among the 6 patient samples across all time points, representing a total of 18 samples.

NK1 cells $\left(\mathrm{CD}_{4} 5^{+}, \mathrm{CD} 6 \mathrm{a}^{-}, \mathrm{CD}^{-}, \mathrm{CD} 19^{-}, \mathrm{CD} 20^{-}, \mathrm{CD} 14^{-}, \mathrm{CD} 11 \mathrm{c}^{-}, \mathrm{CD} 56^{-}, \mathrm{CD}^{+} 6^{+}\right)$and increased DCs, along with increasing FLT-3L and GM-CSF levels. Combined, these data indicate a shift from immune suppression to immune activation after surgery in patient 2 (IDH1 mutant) compared with patient 4 (IDH1 WT), who maintained and enhanced their immune suppressive status (Supplemental Figure 12B and Supplemental Figure 13, A and B). Based on these findings, we performed tSNE plots of MDSCs over time, where patients 2,6, and 7, who had a good prognosis and decreasing MDSCs over time, were compared with patients 4 and 5 , who had increasing MDSCs with poor prognosis, with the hypothesis that the addition or subtraction of patient 2 (IDH1 mutant) would skew results; no such skewing was observed (Supplemental Figure 12A).

CyTOF analysis identifies changes in the immune system over time. To quantify which immune cell populations changed over time, each population of immune cells was individually assessed. This analysis indicated that M-MDSCs, DCs, double-positive T cells, $\mathrm{CD}^{+} \mathrm{T}$ cells, and a mixed population of cells expressing a combination of $\mathrm{B}$ and $\mathrm{T}$ cell markers were significantly altered during disease progression (Figure 3 ). While B cells were significantly reduced compared with baseline, CD8 ${ }^{+} \mathrm{T}$ cells, DCs, and M-MDSCs were significantly increased at 2 months after surgery compared with baseline (Figure 3 ). While the increased CD8 ${ }^{+} \mathrm{T}$ cells and DCs are indicative of an antitumor immune response, there was also a reduction in B cells and an increase in immunosuppressive M-MDSCs and double-positive T cells, which are controversial and could be immunosuppressive or antitumor, depending on the context (45). Strong systemic immunosuppression was thus induced by the tumor. These data indicate that specific cell populations, including M-MDSCs, change during disease progression.

CyTOF analysis of the CD33+ myeloid population of patients identifies increased DCs associated with patients exhibiting a good prognosis. Based on our interest in the myeloid compartment and the observation that intratumoral MDSCs were associated with a poor prognosis while CD33 myeloid cells were associated with a good prognosis, we utilized CyTOF to interrogate the peripheral blood myeloid population over time in 3 patients with increasing MDSCs and a poor prognosis and 2 patients with decreasing MDSCs and a good prognosis. MDSC levels were previously assessed by flow cytometry (Figure 2) over time, and we observed that patients 6 and 7 had decreasing MDSCs over time and a good prognosis (survival > 20 months), while patients 4, 5, and 9 had increasing MDSCs over time and a poor prognosis (survival < 20 months) (Supplemental Figure 11). To 

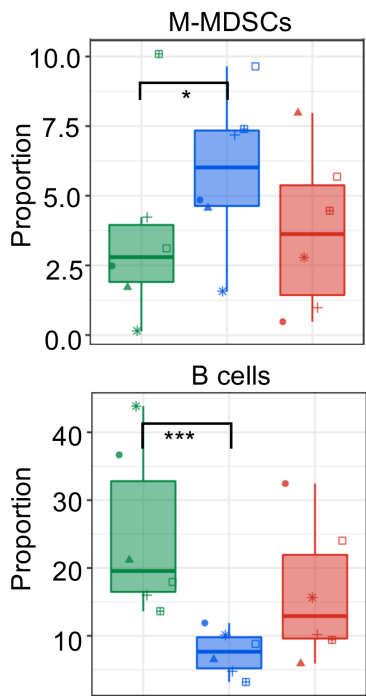

T/B cell marker mix
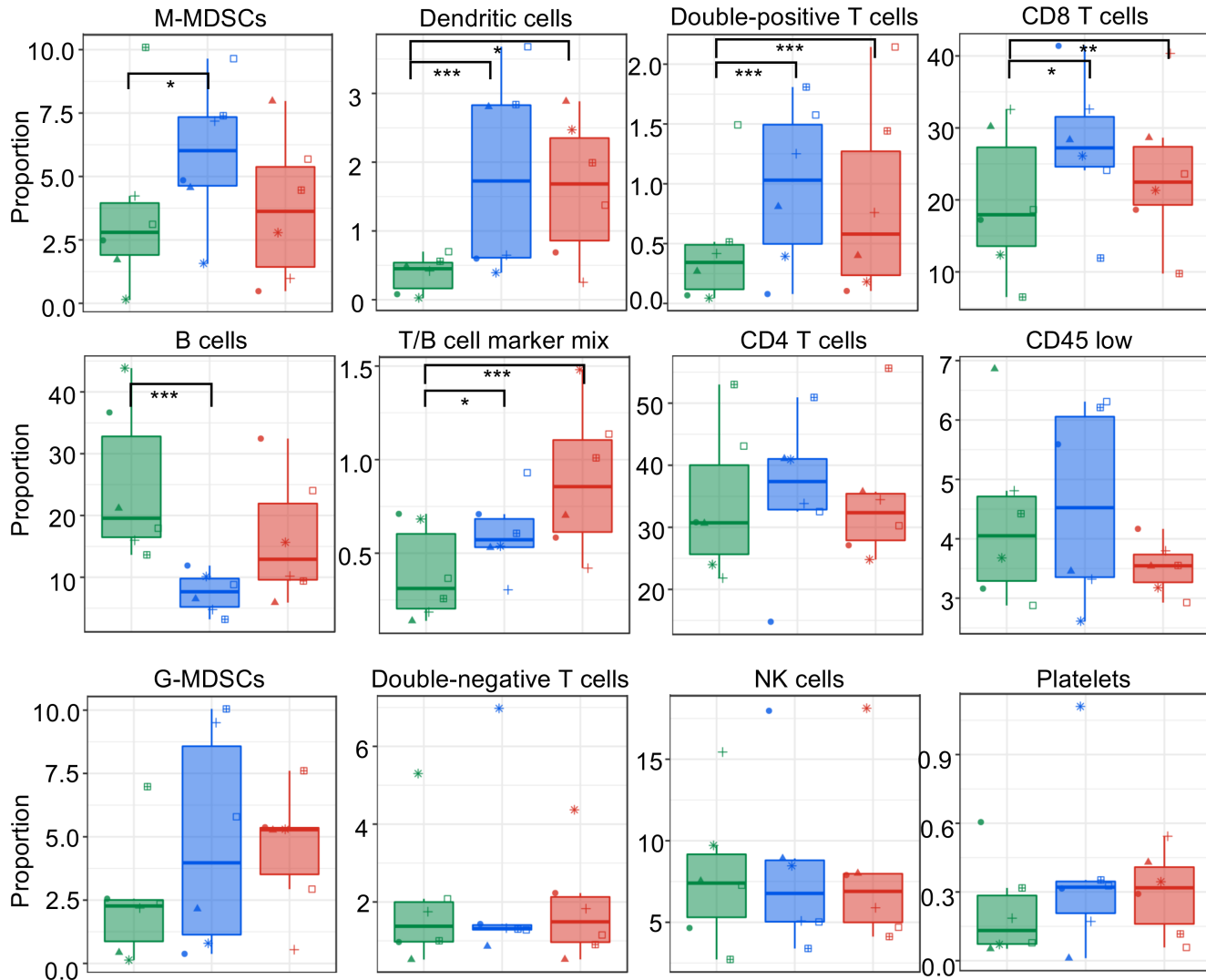
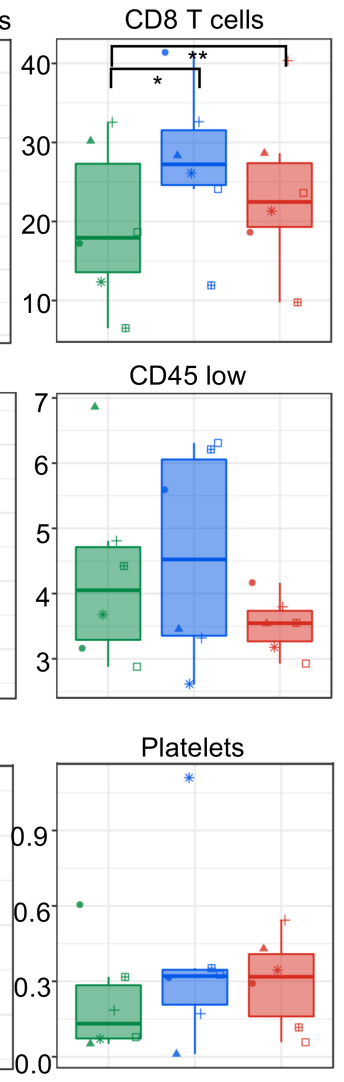

\begin{tabular}{|l|}
\hline KEY \\
Patient \\
• $\mathrm{P} 2$ \\
A $\mathrm{P} 3$ \\
* $\mathrm{P} 4$ \\
$+\mathrm{P} 6$ \\
⿴囗十 $\mathrm{P} 7$ \\
口 $\mathrm{P} 9$ \\
\hline
\end{tabular}

Figure 3. СутOF identifies immune cell populations that are significantly altered during disease progression. Using 12 immune cell populations that were identified in an unbiased manner from baseline (green), time point 1 (blue), and time point 2 (red) samples for $n=6$ newly diagnosed GBM patients were examined via 2-tailed Student's $t$ test to compare baseline to time points 1 and 2 . Each patient is indicated by the symbol identified in the key to the right. Statistics were determined by comparing baseline to each time point using linear models of the data with 2-tailed $t$ test comparisons and Benjamini-Hochberg to adjust to control for multiple comparisons. ${ }^{*} P<0.05,{ }^{* *} P<0.001,{ }^{* * *} P<0.0001$. Graphs represent data sets as median with first and third quartiles.

investigate if whether changes occurred within the myeloid compartment over time, tSNE plots were used to view any alterations in the $\mathrm{CD} 33^{+}$populations at baseline, time point 1 , and time point 2 in each group (Figure $4 \mathrm{~B})$. These tSNE plots identified distinct differences in the myeloid cell populations over time between patients with a good prognosis and a poor prognosis. To further determine which markers where changed, a heatmap was used to view the markers expressed on $\mathrm{CD} 33^{+}$myeloid cells as a fold change from baseline (Figure 4C). This analysis identified CD11c and HLA-DR as being increased over time in patients 6 and 7, both of whom had decreasing MDSCs and a good prognosis (Figure 4C). Additionally, patients 4, 5, and 9 had increased CD61 expression on in the myeloid compartment, which has been previously linked to GBM and is involved in leukocyte rolling and adhesion $(46,47)$. This could indicate an increased ability to infiltrate the tumor and suppress the immune system within the tumor microenvironment $(46,47)$.

Immunosuppressive NK2 cells increase with increased MDSCs, while DC levels are inversely correlated with MDSC levels. To gain a more in-depth appreciation of the changes in immune cell populations between patients with decreasing MDSCs and a good prognosis (patients 6 and 7; survival > 20 months) and patients with increasing MDSCs and a poor prognosis (patients 4, 5, and 9; survival < 20 months) (Supplemental Figure 7), we focused on immunosuppressive MDSCs, DCs (Figures 3 and 4), and NK cells, which have an established role in the antitumoral immune response (12, 13, 22, 48, 49). Patients 6 and 7 showed decreases in MDSCs over time that were confirmed by manual gating of CyTOF data, where previous identification of this phenomenon was by flow cytometry and unbiased CyTOF analysis (Figure 5A). Additionally, immunosuppressive NK2 cells (CD45 ${ }^{+}, \mathrm{CD}_{66 a^{-}}, \mathrm{CD}^{-}$, $\mathrm{CD}^{-} 9^{-}, \mathrm{CD} 20^{-}, \mathrm{CD} 14^{-}, \mathrm{CD} 11 \mathrm{c}^{-}, \mathrm{CD}^{2} 6^{+}, \mathrm{CD} 16^{-}$) were found to be increased along with MDSCs (Figure $5 \mathrm{~B}$ ) (50). Following this finding, the fold change in the ratio of NK2/NK1 cells was examined and confirmed the specific increase of immunosuppressive NK2 cells with no change in antitumoral 
A

$$
\begin{aligned}
& \text { Good prognosis } \\
& \text { ( Patient } 6,7 \text { ) }
\end{aligned}
$$

Poor prognosis (Patient 4,5,9)
MDSC Decrease Survival $>20$ months

MDSC Increase Survival $<20$ months

B Change in Myeloid Populations over time

Increasing MDSCs $\mathrm{P} 4,5,9$

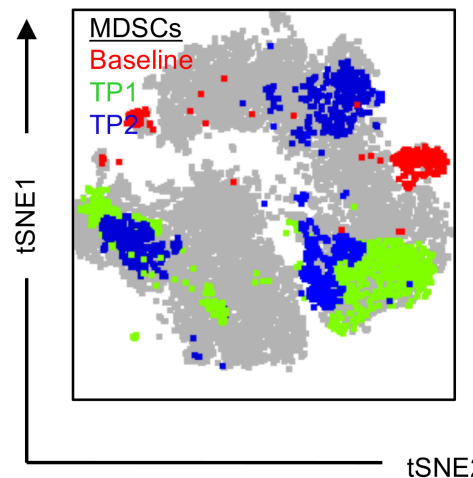

Decreasing MDSCs P6,7

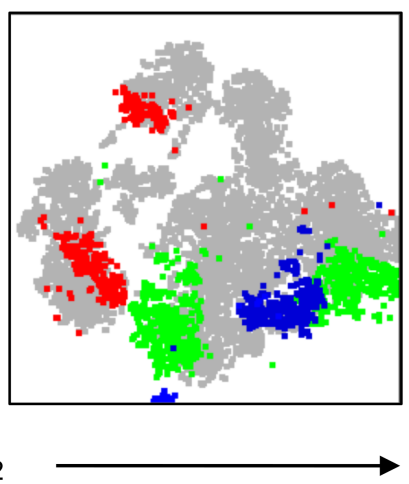

C

Increasing MDSCs Decreasing MDSCs $P 4,5,9$ P6,7

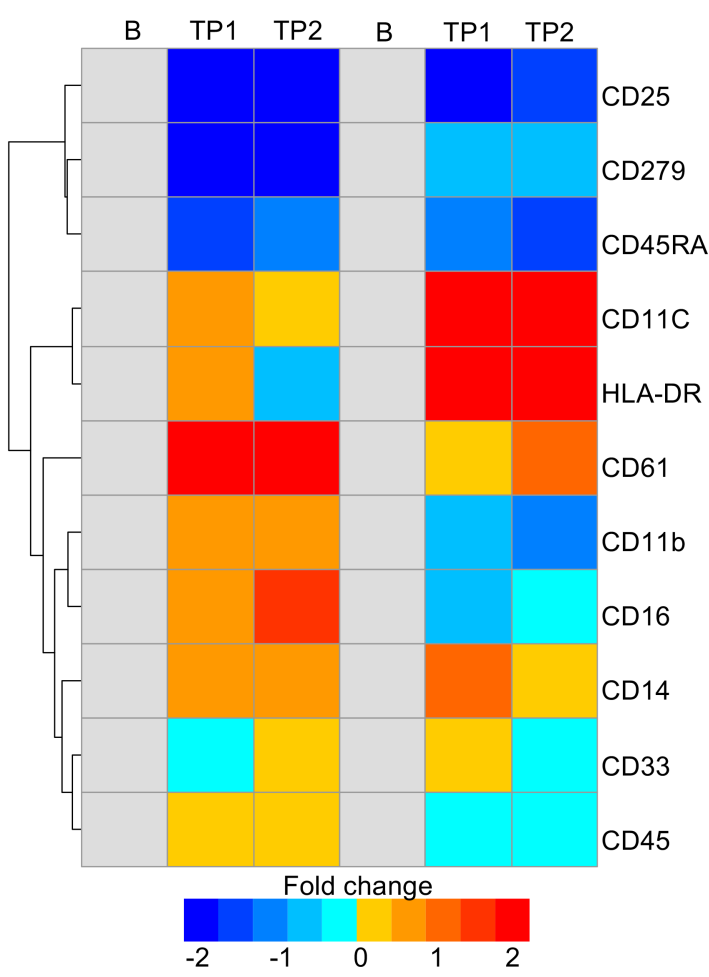

Figure 4. In-depth CyTOF analysis of patients with differing prognoses identifies shifts in MDSCs and other immune populations. (A) Schematic representation of the 2 groups used for in-depth manual gating analysis (patients 6 and 7 vs. patients 4, 5, and 9). Patients 6 and 7 had a good prognosis (survival >20 months after diagnosis) and decreasing MDSCs as identified by flow cytometry, while patients 4, 5, and 9 had a poor prognosis (survival <20 months after diagnosis) and increasing MDSCs as identified by flow cytometry. (B) tSNE analysis of CD33+ myeloid cells over time at baseline, time point 1 , and time point 2, where manually gated myeloid cells were overlaid and colored according to their time points (baseline, red; time point 1 , green; time point 2, blue). (C) Myeloid cells of patients in both groups were examined for fold change in myeloid markers from baseline using the CyTOF panel.

NK1 cells (CD45 ${ }^{+}, \mathrm{CD}_{66 a^{-}}, \mathrm{CD}^{-}, \mathrm{CD} 19^{-}, \mathrm{CD}^{-} 0^{-}, \mathrm{CD}^{-} 4^{-}, \mathrm{CD} 11 \mathrm{c}^{-}, \mathrm{CD}^{-} 6^{-}, \mathrm{CD}^{+} 6^{+}$) (Figure 5C) (51, 52). Based on the identification of a possible switch from MDSCs to DCs identified in patients with decreases in MDSCs (Figure 4), we analyzed the ratio of MDSCs/CD11 $\mathrm{c}^{+}$cells (Figure 5D). Consistent with previous findings, DCs increased as MDSCs reduced over time in patients 4,5 , and 9. The reduction in MDSCs combined with increased DCs indicates the potential differentiation of MDSCs into DCs $(53,54)$. To determine whether environmental conditions were favorable for MDSC differentiation, a 65-plex flow cytometry-based cytokine array was applied to serum samples matching the blood samples at baseline, $t_{1}$ (2 months), and $t_{2}$ (final sampling time) (Supplemental Figure 14) (55). Additional patients were examined via cytokine array, which revealed a clear signature of cytokine expression that differed between LGG and GBM patients (Supplemental Figure 14).

CyTOF analysis of LGG patients reveals alterations in DCs and NK cells, similar to those of a GBM patient with a favorable prognosis. Based on the distinct immune activation statuses found between patients with good and poor prognoses, we compared 6 GBM patients with 3 LGG patients at diagnosis using samples on which we performed baseline CyTOF analyses (LGG1, IDH1 mutant; LGG2, IDH1 WT; LGG3, IDH1 WT). Although MDS revealed no clear difference between patients with GBM and LGG, tSNE analysis showed shifts in immune cell populations between GBM and LGG patients (Supplemental Figure 15). Further identification of the clusters within the tSNE and a quantification of immune cell changes revealed that the only significantly altered immune cell populations were DCs and NK cells, both of which were higher in LGG patients than in GBM patients (Figure 6, A and B, and Supplemental Figures 15-17). These results were consistent with our longitudinal study finding that a higher frequency of NK cells and DCs is associated with a favorable prognosis and suggest that such GBM patients have an immune landscape similar to that of LGG patients. 
A

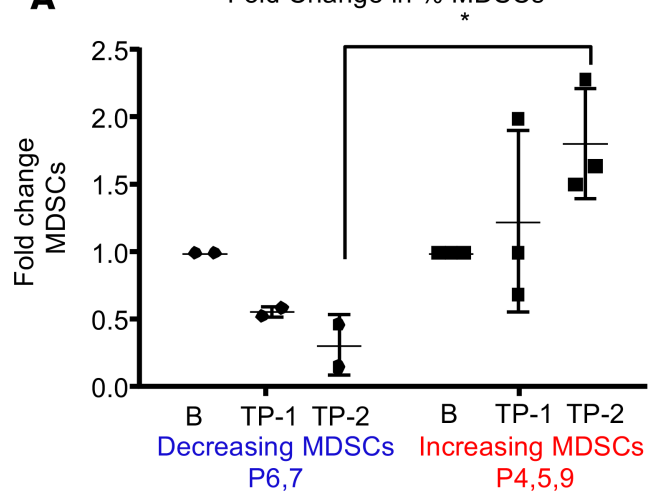

C Fold change NK2/NK1 ratio

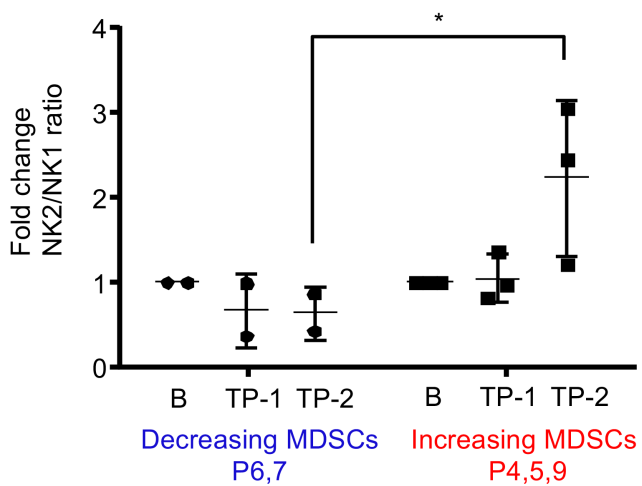

B

Fold Change NK2 cells

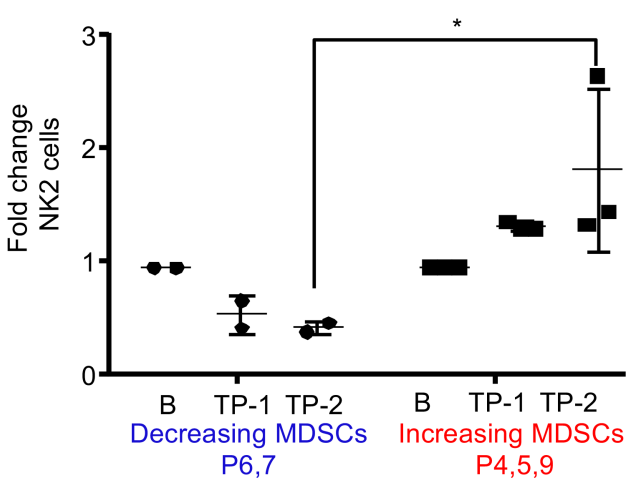

D

Fold Change MDSC/CD11c ratio

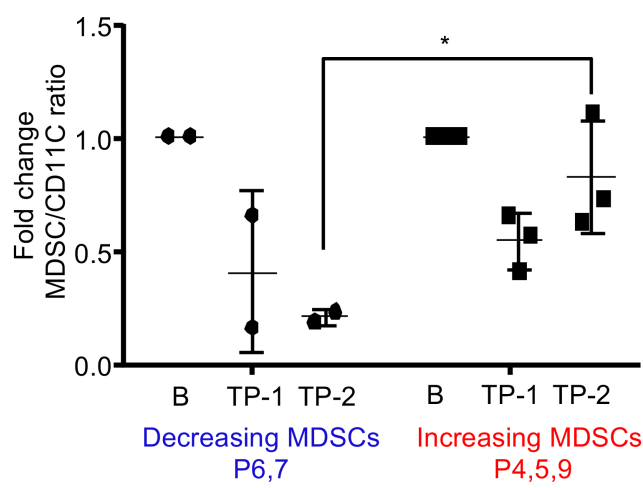

Figure 5. DCs and antigen-presenting cells are increased in a patient with a good prognosis. (A-D) Manual gating of MDSCs, NK2, NK1, and DC populations from the decreasing MDSCs group and the increasing MDSCs group at baseline (B), time point 1 (1), and time point 2 (2), where $B$ is baseline, 1 is 2 months after diagnosis, and 2 is the final time point collected. Graphs represent data as mean \pm SD.

\section{Discussion}

The correlation between peripheral antitumoral immune response and tumoral immune response have been of great interest; however, the identification of the peripheral immune status of GBM patients compared with that of patients with other types of brain tumors has not been comprehensively assessed. As the field of tumor immunotherapy progresses, it is vital to determine how the systemic immune response is altered under various tumor diagnoses, as past experiences have revealed that 1 drug does not work for all patients with the same disease, and it appears that immunotherapies are encountering a similar roadblock (56). To identify new immunotherapeutic approaches or to enhance the efficacy of existing ones, we must first understand the peripheral immune landscape that is altered by the tumor and then ask how the drug of interest impacts that landscape (57).

For GBM, patients have a skewed immune system with increased immunosuppression. This was recently highlighted by a report showing that T cell levels in GBM patients are dereased to the level of patients with AIDS (19). However, studies typically focus on only 1 or 2 immune cell types of interest and do not examine the immune landscape as a whole or the immune response relative to that of patients with other brain tumors. Here, we have developed a CyTOF panel to provide an understanding of the immune system as a whole and to predict how immune-modulating therapies may impact the antitumoral immune response of patients (58). This understanding will aid in the investigation of future drugs in an unbiased manner by analyzing immune cell types implicated in immunosuppression and activation within GBM. We hypothesized that MDSCs are increased in GBM patients compared with patients with other types of brain tumors, based on the increased malignancy of GBM, and that the systemic immune response to GBM may differ over time among patients based on their prognosis and diagnosis. Our findings support this hypothesis and reveal that GBM patients with a more favorable prognosis exhibit decreased MDSCs and increased DCs, suggesting that MDSC differentiation may be associated with an increase in immune activation and, thus, a decrease in GBM growth.

Through these studies, we found that immunosuppressive MDSCs are elevated in high-grade glial malignancies and in nonglial malignancies with brain metastases, while suppressive $\mathrm{T}$ cell populations 
A

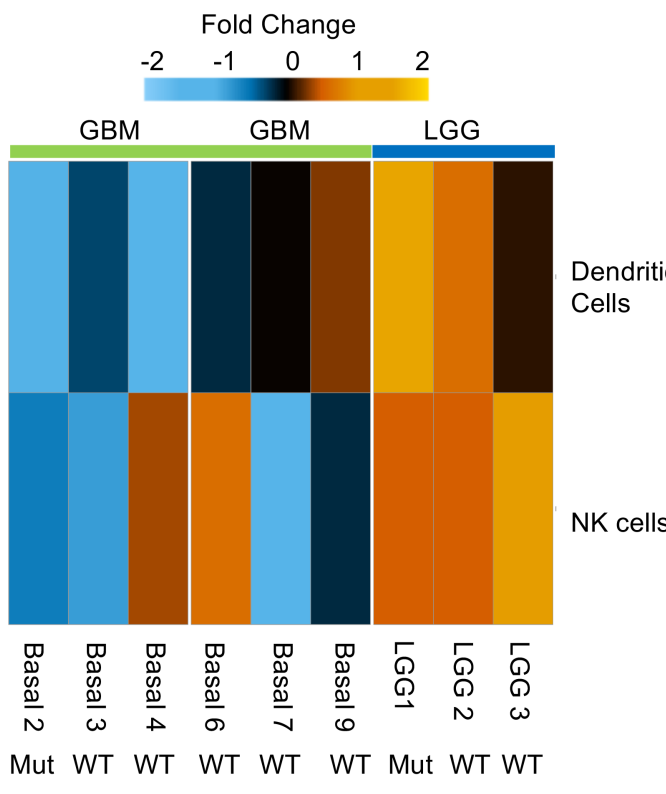

B

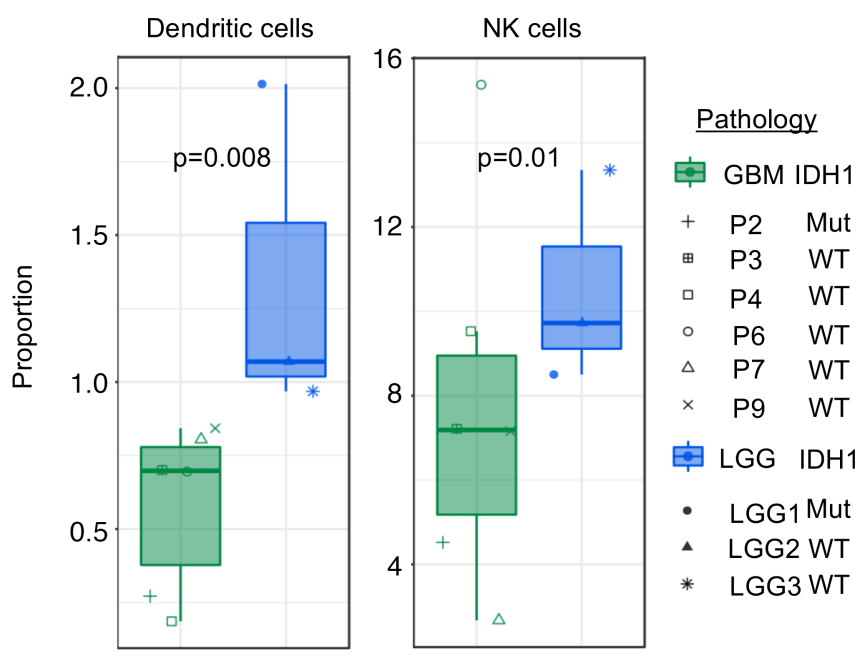

IDH1

status

Figure 6. Compared with LGG patients, GBM patients have reduced antigen-presenting cells and NK cells, which is indicative of a reduced antitumoral response. (A) Unbiased clustering of CyTOF data identifies NK cells and DCs as different between patients with LGG $(n=3)$ and GBM $(n=6)$ at baseline as organized by hierarchical clustering. (B) Quantification of NK cells and DCs in 6 GBM patients and 3 LGG patients at baseline using the $t$ test. Graphs represent data sets as median with first and third quartiles.

were not increased, as previously reported $(29,59,60)$. This is important, as many therapeutic strategies currently under investigation for GBM aim to activate T cells, as opposed to targeting the immunosuppressive cell types induced by the tumor (3). Of interest, we also found that steroid use trends toward being a significant predictor of MDSC levels $(P=0.06)$ in a univariable linear model. It is possible that this study was not sufficiently powered to determine the role of steroid use, but evidence is mounting that steroids alter the myeloid populations of GBM patients toward increased immune suppression, strengthening the idea of targeting these cells in GBM patients, given that they all receive corticosteroids at the time of surgery. While systemic immunosuppression was observed in these studies, we also observed immunosuppression intratumorally, where MDSCs correlated with overall survival. This observation was made using matched primary and recurrent tumor-resection samples, where elevated levels of $\mathrm{CD} 3^{+}$myeloid cell levels at recurrence correlated with a good prognosis, while infiltration of a specific subtype of myeloid cells, MDSCs, into the tumor microenvironment correlated with poor prognosis. These findings align with the genomic analysis of the immune landscape of IDH1 WT and mutant gliomas previously identified (42). Based on these findings, future studies could be performed to confirm the utility of MDSCs as a biomarker of disease malignancy and progression in brain tumor patients.

To gain an understanding into how patients' immune systems change over time with disease progression, a CyTOF panel of 25 immune markers was developed that identified alterations in immune activation status and immunosuppression as a function of time. Specifically, while DCs and CD8 ${ }^{+} \mathrm{T}$ cells increased over time, there was also a corresponding increase in immunosuppressive M-MDSCs and a decrease in B cells. The increase in DCs is of particular interest because it has been shown that DCs from the circulation are more effective at activating an antitumoral immune response than resident antigen presenting cells such as microglia (61-63). This phenomenon of immune recognition without an antitumoral immune response has also been observed in clinical trials of immunotherapies (63). The associations identified between GBM patients and their immunosuppression status with increasing MDSCs paves the way for future studies to combine anti-MDSC therapy with immune checkpoint therapies to enhance efficacy. Based on the differences noted between LGG patients and GBM patients by multiparameter flow cytometry analysis, CyTOF was performed at baseline for LGG and GBM patients. DC and NK cell levels were higher in LGG patients compared with GBM patients, which could indicate that LGG patients are primed for an antigen response prior to surgery and are, thus, better able to mount an antitumor immune response to some degree. On the basis that MDSCs have the 
ability to differentiate into DCs $(24,54)$, the data here suggest that LGG favor MDSCs maturing into DCs and that high-grade tumors favor MDSCs remaining as MDSCs. Future studies targeting differentiation pathways could be pursued as a possible avenue to enhance the antitumoral immune response and increase the efficacy of immune activating therapies.

\section{Methods}

Study design. We sought to determine the relative frequency of MDSCs in GBM patients compared with patients with other primary and secondary malignant and benign brain tumors and, using CyTOF technology, to determine how the immune system of GBM patients is altered. Blood samples from a total of 259 patients were collected from brain tumor patients entering the Cleveland Clinic for treatment under Cleveland Clinic IRB 2559. Patients were grouped by their diagnoses into the categories benign, nonglial malignancy, grade I/II, grade III, grade IV, and other, as outlined in Supplemental Figure 2. Additionally, a cohort of 6 newly diagnosed GBM patients was enrolled in a blood collection study where blood samples were drawn every 2 months, with samples stored for general use by the Rose Ella Burkhardt Brain Tumor and Neuro-Oncology Center. Patient data was blinded from the researchers by the Rose Ella Burkhardt Brain Tumor and Neuro-Oncology Center through the generation of a deidentified numbering system. Multiparameter flow cytometry and CyTOF panels were designed with MDSC and $\mathrm{T}$ cell populations in mind based on their relevance to GBM and previous identification within GBM (Supplemental Figure 1). Additionally, tumor tissue from 22 patients was retrospectively investigated from Odense University Hospital. All patients had been diagnosed with primary GBM between 2007 and 2015 and had not received any treatment prior to initial surgery. Following initial surgical resection, all patients received radiotherapy and chemotherapy. All patients experienced tumor recurrence within 31 months (mean progression-free survival, 13.3 months; range, 4.9-30.4 months), and the time period between initial and surgery resection was 15.2 months, on average (range, 5.1-37.4 months; Supplemental Figure 3). Four patients were diagnosed with recurrent GBM of the subtype gliosarcoma, and the remaining 18 were diagnosed with recurrent GBM.

Flow cytometry. Peripheral blood samples were analyzed to determine MDSC and T cell populations in GBM patients over time, as well as in LGG patients, and were carried out in accordance with an approved Cleveland Clinic Foundation IRB protocol. Upon arrival, samples were processed through a Ficoll gradient in Ficoll-Paque PLUS and SepMate (Stemcell Technologies) tubes before being suspended in freezing medium for storage. Samples were stained with live/dead UV stain (Invitrogen) and then blocked in FACS buffer (PBS, 2\% BSA) containing FcR blocking reagent at 1:50 (Miltenyi Biotec) for 15 minutes. After live/dead staining and blocking, antibody cocktails (Supplemental Figure 1) were incubated with samples on ice for 25 minutes before being washed and suspended in FACS buffer. Cell populations were analyzed using an LSRFortessa (BD Biosciences), and populations were separated and quantified using FlowJo software (Tree Star Inc.). Gating methods for MDSCs were performed following standardized gating strategies previously described and outlined in Supplemental Figure 1, where MDSCs are marked by CD $11 b^{+}$, $\mathrm{CD} 33+$, and HLA-DR ${ }^{-/ 10}$ and can then be further subdivided into granulocytic MDSCs $\left(\mathrm{CD} 15^{+}\right)$and M-MDSCs $\left(C D 14^{+}\right)(64)$. Tregs were gated as $\mathrm{CD}^{+}, \mathrm{CD}^{+}, \mathrm{CD}^{+} 5^{+}$, and $\mathrm{CD} 127^{-}$, as previously described (65). $\mathrm{CD}^{+} \mathrm{T}$ cells were gated on $\mathrm{CD}^{+}, \mathrm{CD}^{+}$, and $\mathrm{CD}^{-}$, which were then determined to be activated by expression of the degranulation marker CD107a (66).

Immunofluorescence. Fresh tissue biopsies were fixed in $4 \%$ neutral buffered formalin and embedded in paraffin. Sections $(3 \mu \mathrm{m})$ were used for triple immunofluorescence staining, which was performed on a Dako Autostainer Universal Staining System (Dako). Heat-induced epitope retrieval (HIER) was performed in a buffer solution consisting of $10 \mathrm{mmol} / 1$ Tris base and $0.5 \mathrm{mmol} / 1 \mathrm{EGTA}, \mathrm{pH} 9$, followed by blocking of endogen peroxidases with hydrogen peroxide. Sections were then incubated for 60 minutes with a primary antibody against CD33 (1:200, NCL-L, Novocastra), and the antigen-antibody complex was detected using CSA II Biotin-free Tyramide Signal Amplification System kit (Dako) with fluorescein as the fluorochrome. After a second round of HIER followed by endogenous peroxidase quenching was performed, sections were incubated with an anti-HLA-DR antibody (1:200, CR3/43, Dako) for 60 minutes, and Tyramide Amplification Signal Cyanine 5 (TSA-Cy5, Perkin Elmer) was used as the detection system. Sections were then washed and incubated with an anti-IBA1 antibody (1:300, 019-19741, Wako Pure Chemical Industries) for 60 minutes, followed by detection with a goat antirabbit Alexa 350 secondary antibody (1:100, A-110461, Invitrogen). Coverslips were mounted using 
VECTASHIELD Mounting Medium (VWR International). Omission of primary antibodies served as negative control. Fluorescent imaging and quantitation were carried out using the Visiopharm integrated microscope and software module (Visiopharm) consisting of a Leica DM6000B microscope connected to an Olympus DP72 1.4 Mega Pixel CCD camera (Olympus) using DAPI (Omega XF06, Omega Optical), FITC (Leica), and cyanine-5 (Omega XF110-2, Omega Optical) filters. Super images were acquired at $1.25 \times$ magnification using brightfield microscopy. Next, sampling regions were manually outlined. Sample images were collected using systematic uniform random (meander) sampling at 20x magnification with a minimum of 5 images per tumor. Images were reviewed to ensure that no artifacts or blurring were present. Images were then analyzed to quantify the amount of MDSCs in each tumor using a threshold-based algorithm developed in the Visiopharm software module. CD33 was used as an inclusion marker, and the algorithm was designed to identify the entire CD $33^{+}$area within the total tumor area. The $\mathrm{CD} 33^{+}$area was then subdivided into IBA $1^{+}$and $\mathrm{IBA} 1^{-}$. The $\mathrm{CD} 33^{+} \mathrm{IBA} 1^{+}$area was then separated into 3 areas based on the intensity of HLA-DR staining: (a) CD $33^{+} \mathrm{IBA} 1^{+} \mathrm{HLA}-\mathrm{DR}{ }^{-}$ area, (b) $\mathrm{CD}_{3} 3^{+} / \mathrm{IBA}^{+} / \mathrm{HLA}-\mathrm{DR}^{10}$ area, and (c) $\mathrm{CD} 33^{+} \mathrm{IBA}^{+} \mathrm{HLA}-\mathrm{DR} \mathrm{R}^{\text {hi }}$ area. From these areas, 7 area fractions were calculated: (a) the $\mathrm{CD}_{3} 3^{+}$area of the total tumor area, (b) the area of MDSCs with no HLA-DR expression within the $\mathrm{CD}_{3} 3^{+}$area, (c) the area of MDSCs with low HLA-DR expression within the $\mathrm{CD}_{3} 3^{+}$area, (d) the total MDSC area (i.e., both HLA-DR ${ }^{-}$and HLA-DR ${ }^{10}$ ) within the CD33 ${ }^{+}$ area, (e) the area of MDSCs with no HLA-DR expression within the total tumor area, (f) the area of MDSCs with low HLA-DR expression within the total tumor area, and (g) the total MDSC area (i.e., both HLA-DR ${ }^{-}$and HLA-DR ${ }^{10}$ ) within the total tumor area. All areas were analyzed for their association with survival and outlined in Supplemental Figure 3.

CyTOF. Mass cytometry was performed in collaboration with the UCLA Jonsson Comprehensive Cancer Center (JCCC) and Center for AIDS Research Flow Cytometry Core Facility on a Fluidigm Helios CyTOF system. All antibodies were validated within the core, and those listed with heavy metal tags are listed in Supplemental Figure 7 and determined to be nonoverlapping by Maxpar Panel Designer Panel Wheel (Fluidigm). Cell were labeled with cisplatin (Cell-ID Cisplatin; Fluidigm), a cocktail of metal-conjugated surface marker antibodies, and iridium (Cell-ID Intercalator; Fluidigm) using reagents and protocols provided by Fluidigm. Before analysis, populations were cleaned by removing debris and dead cells before analysis (Supplemental Figure 7). Samples were analyzed from 6 GBM patients at 3 time points for each patient (baseline, 2 months after recurrence, and final sample collected). Three patients from this group had a good prognosis, as denoted by a survival $>20$ months after primary resection, and were still surviving, while 3 patients had a poor prognosis as denoted by a survival $<20$ months. Additionally, 3 LGG patients were analyzed at baseline for the comparison of baseline samples.

CyTOF analysis. Prior to running CyTOF samples through data analysis, FCS files were normalized between runs using beads and the Nolan lab bead normalizer package (67). The most current CyTOF data analysis tools were used for data analysis, including multidimensional analysis with $\mathrm{R}$ following methods described by Nowicka et al. (40). Additionally, FlowSOM analysis of CyTOF data was performed to identify changes in cell populations in an unbiased manner (68). In a biased approach, CyTOF data was also analyzed using FlowJo software (Tree Star Inc.) as outlined in Supplemental Figure 7.

Cytokine analysis. Cytokine analysis of patient samples was performed using a flow cytometry-based 65-plex cytokine array (Eve Technologies) (Supplemental Figure 14).

Statistics. R version 3.4.4 was used in data analyses. The R function $1 \mathrm{~m}()$ was used to model cell percentages of live cells as linear combinations of clinical covariates; as all values of such percentages were below $15 \%$, saturation of percent was not a concern. Stata (StataCorp) was used to compute log-rank test $P$ values and Cox proportional hazard model parameter $P$ values.

Study approval. Blood samples from $n=259$ brain tumor patients were collected at the Cleveland Clinic when undergoing treatment via the Rose Ella Burkhardt Brain Tumor and Neuro-Oncology Center at the Cleveland Clinic in accordance with IRB 2559. All GBM tumor tissue samples were reevaluated according to WHO 2016 guidelines. Use of tissue was approved by the official Danish ethical review board (the Regional Scientific Ethical Committee of the Region of Southern Denmark), which approved the use of human glioma tissue (permission J. No. S-2011 0022). Use of the tissue was not prohibited by any of the patients according to the Danish Tissue Application Register. 


\section{Author contributions}

TJA, AGA, MAV, and JDL provided conceptualization and design; TJA, AGA, MDS, DB, JV, ES, EEMH, MS, JSH, MM, PH, MMG, and CAW performed the experiments; TJA, AGA, MDS, JV, GRO, MSA, TR, HIK, BWK, MAV, and JDL analyzed the data; TJA, DB, EEMH, MAV, and JDL wrote the manuscript; HIK, BJW, MAV, and JDL provided financial support; and all authors provided final approval of the manuscript.

\section{Acknowledgments}

We thank the patients treated at the Rose Ella Burkhardt Brain Tumor and Neuro-Oncology Center and Odense University Hospital for donation of their blood samples for this study. We thank the staff of the Rose Ella Burkhardt Brain Tumor and Neuro-Oncology Center and Odense University Hospital for their collaboration acquiring samples. We thank the members of the Lathia laboratory and Ofer Reizes and his laboratory for insightful discussion and constructive comments on the manuscript. We thank Joseph Gerow and Eric Schultz for flow cytometry assistance. We thank the Janis V. Giorgi Flow Cytometry Core Laboratory (UCLA) for their assistance with CyTOF experiments. We thank Amanda Mendelsohn and the Center for Medical Art and Photography at the Cleveland Clinic for providing illustrations. This work was funded by a NIH grant (F31 NS101771, TJA), the Sontag Foundation (JDL), Blast GBM (JDL, MAV), the Cleveland Clinic VeloSano Bike Race (JDL, MAV), B*CURED (JDL, MAV), and Case Comprehensive Cancer Center (JDL, MAV). Flow cytometry was performed in the UCLA JCCC and Center for AIDS Research Flow Cytometry Core Facility, which is supported by NIH awards P30 CA016042 and 5P30 AI028697. The purchase of the Helios mass cytometer that was used in this work was, in part, supported by the funds provided by the James B. Pendleton Charitable Trust. HIK and AGA were supported by the Dr. Miriam and Sheldon G Adelson Medical Research Foundation.

Address correspondence to: Justin D. Lathia, Department of Cellular and Molecular Medicine, Lerner Research Institute, 9500 Euclid Avenue, NC10, Cleveland, Ohio 44195, USA. Phone: 216.445.7475; Email: lathiaj@ccf.org. Or to: Michael A. Vogelbaum, Rose Ella Burkhardt Brain Tumor and Neuro-Oncology Center, 9500 Euclid Avenue, ND40, Cleveland, Ohio 44195, USA. Phone: 216.445.5522; Email: vogelbm@ccf.org.

1. Stupp R, et al. Effects of radiotherapy with concomitant and adjuvant temozolomide versus radiotherapy alone on survival in glioblastoma in a randomised phase III study: 5-year analysis of the EORTC-NCIC trial. Lancet Oncol. 2009;10(5):459-466.

2. Farkona S, Diamandis EP, Blasutig IM. Cancer immunotherapy: the beginning of the end of cancer? BMC Med. $2016 ; 14: 73$.

3. Lim M, Xia Y, Bettegowda C, Weller M. Current state of immunotherapy for glioblastoma. Nat Rev Clin Oncol. 2018;15(7):422-442.

4. Peggs KS, Quezada SA. Ipilimumab: attenuation of an inhibitory immune checkpoint improves survival in metastatic melanoma. Expert Rev Anticancer Ther. 2010;10(11):1697-1701

5. Yang I, et al. CD8+ T-cell infiltrate in newly diagnosed glioblastoma is associated with long-term survival. J Clin Neurosci. 2010;17(11):1381-1385.

6. Rossi ML, Hughes JT, Esiri MM, Coakham HB, Brownell DB. Immunohistological study of mononuclear cell infiltrate in malignant gliomas. Acta Neuropathol. 1987;74(3):269-277.

7. Heimberger $\mathrm{AB}$, et al. Incidence and prognostic impact of FoxP3+ regulatory T cells in human gliomas. Clin Cancer Res. 2008;14(16):5166-5172.

8. Li B, et al. Landscape of tumor-infiltrating T cell repertoire of human cancers. Nat Genet. 2016;48(7):725-732

9. Donson AM, et al. Increased immune gene expression and immune cell infiltration in high-grade astrocytoma distinguish longterm from short-term survivors. J Immunol. 2012;189(4):1920-1927.

10. Doucette $\mathrm{T}$, et al. Immune heterogeneity of glioblastoma subtypes: extrapolation from the cancer genome atlas. Cancer Immunol Res. 2013;1(2):112-122.

11. Bingle L, Brown NJ, Lewis CE. The role of tumour-associated macrophages in tumour progression: implications for new anticancer therapies. J Pathol. 2002;196(3):254-265.

12. Raychaudhuri B, et al. Myeloid derived suppressor cell infiltration of murine and human gliomas is associated with reduction of tumor infiltrating lymphocytes. J Neurooncol. 2015;122(2):293-301.

13. Raychaudhuri B, et al. Myeloid-derived suppressor cell accumulation and function in patients with newly diagnosed glioblastoma. Neuro-oncology. 2011;13(6):591-599.

14. Mirzaei R, Sarkar S, Yong VW. T Cell Exhaustion in Glioblastoma: Intricacies of Immune Checkpoints. Trends Immunol. 2017;38(2):104-115.

15. Gielen PR, et al. Elevated levels of polymorphonuclear myeloid-derived suppressor cells in patients with glioblastoma highly express S100A8/9 and arginase and suppress T cell function. Neuro-oncology. 2016;18(9):1253-1264.

16. Charoentong $P$, et al. Pan-cancer Immunogenomic Analyses Reveal Genotype-Immunophenotype Relationships and Predictors of Response to Checkpoint Blockade. Cell Rep. 2017;18(1):248-262.

17. Rizvi NA, et al. Cancer immunology. Mutational landscape determines sensitivity to PD-1 blockade in non-small cell lung cancer. Science. 2015;348(6230):124-128. 
18. Carpentier AF, Meng Y. Recent advances in immunotherapy for human glioma. Curr Opin Oncol. 2006;18(6):631-636.

19. Chongsathidkiet $\mathrm{P}$, et al. Sequestration of $\mathrm{T}$ cells in bone marrow in the setting of glioblastoma and other intracranial tumors. Nat Med. 2018;24(9):1459-1468.

20. Filley AC, Henriquez M, Dey M. Recurrent glioma clinical trial, CheckMate-143: the game is not over yet. Oncotarget. 2017;8(53):91779-91794.

21. Carson MJ, Doose JM, Melchior B, Schmid CD, Ploix CC. CNS immune privilege: hiding in plain sight. Immunol Rev. 2006;213:48-65.

22. Otvos B, et al. Cancer Stem Cell-Secreted Macrophage Migration Inhibitory Factor Stimulates Myeloid Derived Suppressor Cell Function and Facilitates Glioblastoma Immune Evasion. Stem Cells. 2016;34(8):2026-2039.

23. Meyer C, et al. Frequencies of circulating MDSC correlate with clinical outcome of melanoma patients treated with ipilimumab. Cancer Immunol Immunother. 2014;63(3):247-257.

24. Gabrilovich DI, Nagaraj S. Myeloid-derived suppressor cells as regulators of the immune system. Nat Rev Immunol. 2009;9(3):162-174.

25. Highfill SL, et al. Bone marrow myeloid-derived suppressor cells (MDSCs) inhibit graft-versus-host disease (GVHD) via an arginase-1-dependent mechanism that is up-regulated by interleukin-13. Blood. 2010;116(25):5738-5747.

26. Ortega-Gómez A, Perretti M, Soehnlein O. Resolution of inflammation: an integrated view. EMBO Mol Med. 2013;5(5):661-674.

27. Dvorak HF. Tumors: wounds that do not heal-redux. Cancer Immunol Res. 2015;3(1):1-11.

28. Hazeldine J, Lord JM, Belli A. Traumatic Brain Injury and Peripheral Immune Suppression: Primer and Prospectus. Front Neurol. 2015;6:235.

29. Jacobs JF, et al. Regulatory T cells and the PD-L1/PD-1 pathway mediate immune suppression in malignant human brain tumors. Neuro-oncology. 2009;11(4):394-402.

30. Lowther DE, et al. PD-1 marks dysfunctional regulatory T cells in malignant gliomas. JCI Insight. 2016;1(5):85935.

31. Dubinski D, et al. CD4+ T effector memory cell dysfunction is associated with the accumulation of granulocytic myeloid-derived suppressor cells in glioblastoma patients. Neuro-oncology. 2016;18(6):807-818.

32. Tsujikawa T, et al. Quantitative Multiplex Immunohistochemistry Reveals Myeloid-Inflamed Tumor-Immune Complexity Associated with Poor Prognosis. Cell Rep. 2017;19(1):203-217.

33. Tung JW, et al. Modern flow cytometry: a practical approach. Clin Lab Med. 2007;27(3):453-468.

34. Bengsch B, Ohtani T, Herati RS, Bovenschen N, Chang KM, Wherry EJ. Deep immune profiling by mass cytometry links human T and NK cell differentiation and cytotoxic molecule expression patterns. J Immunol Methods. 2018;453:3-10.

35. Subrahmanyam PB, et al. Distinct predictive biomarker candidates for response to anti-CTLA- 4 and anti-PD-1 immunotherapy in melanoma patients. J Immunother Cancer. 2018;6(1):18.

36. Clancy T, Hovig E. Profiling networks of distinct immune-cells in tumors. BMC Bioinformatics. 2016;17(1):263.

37. Ji KA, et al. Resident microglia die and infiltrated neutrophils and monocytes become major inflammatory cells in lipopolysaccharide-injected brain. Glia. 2007;55(15):1577-1588.

38. Shah VO, Civin CI, Loken MR. Flow cytometric analysis of human bone marrow. IV. Differential quantitative expression of T-200 common leukocyte antigen during normal hemopoiesis. J Immunol. 1988;140(6):1861-1867.

39. Tanaka R, et al. Migration of enhanced green fluorescent protein expressing bone marrow-derived microglia/macrophage into the mouse brain following permanent focal ischemia. Neuroscience. 2003;117(3):531-539.

40. Nowicka M, et al. CyTOF workflow: differential discovery in high-throughput high-dimensional cytometry datasets. F1000Res. 2017;6:748.

41. Choi BD, Curry WT. IDH mutational status and the immune system in gliomas: a tale of two tumors? Transl Cancer Res. 2017;6(Supp1 7):S1253-S1256.

42. Amankulor NM, et al. Mutant IDH1 regulates the tumor-associated immune system in gliomas. Genes Dev. 2017;31(8):774-786

43. Zhang $\mathrm{X}$, et al. IDH mutant gliomas escape natural killer cell immune surveillance by downregulation of NKG2D ligand expression. Neuro-oncology. 2016;18(10):1402-1412.

44. Mu L, et al. The IDH1 Mutation-Induced Oncometabolite, 2-Hydroxyglutarate, May Affect DNA Methylation and Expression of PD-L1 in Gliomas. Front Mol Neurosci. 2018;11:82.

45. Overgaard NH, Jung JW, Steptoe RJ, Wells JW. CD4+/CD8+ double-positive T cells: more than just a developmental stage? J Leukoc Biol. 2015;97(1):31-38.

46. Mori S, et al. Direct binding of integrin alphavbeta3 to FGF1 plays a role in FGF1 signaling. J Biol Chem. 2008;283(26):18066-18075.

47. Strell C, Entschladen F. Extravasation of leukocytes in comparison to tumor cells. Cell Commun Signal. $2008 ; 6: 10$.

48. Zamai L, et al. NK cells and cancer. J Immunol. 2007;178(7):4011-4016.

49. Nair S, Dhodapkar MV. Natural Killer T Cells in Cancer Immunotherapy. Front Immunol. 2017;8:1178.

50. Pandya AD, et al. Identification of human NK17/NK1 cells. PLoS One. 2011;6(10):e26780.

51. Kimura MY, Nakayama T. Differentiation of NK1 and NK2 cells. Crit Rev Immunol. 2005;25(5):361-374.

52. Deniz G, et al. Regulatory NK cells suppress antigen-specific T cell responses. J Immunol. 2008;180(2):850-857.

53. Yaddanapudi K, et al. MIF Is Necessary for Late-Stage Melanoma Patient MDSC Immune Suppression and Differentiation. Cancer Immunol Res. 2016;4(2):101-112.

54. Kumar V, Patel S, Tcyganov E, Gabrilovich DI. The Nature of Myeloid-Derived Suppressor Cells in the Tumor Microenvironment. Trends Immunol. 2016;37(3):208-220.

55. Parajuli P, et al. Flt3 ligand and granulocyte-macrophage colony-stimulating factor preferentially expand and stimulate different dendritic and T-cell subsets. Exp Hematol. 2001;29(10):1185-1193.

56. Emens LA, et al. Cancer immunotherapy: Opportunities and challenges in the rapidly evolving clinical landscape. Eur J Cancer. 2017;81:116-129.

57. Thorsson V, et al. The Immune Landscape of Cancer. Immunity. 2018;48(4):812-830.e14.

58. Bendall SC, Nolan GP, Roederer M, Chattopadhyay PK. A deep profiler's guide to cytometry. Trends Immunol. 2012;33(7):323-332.

59. Jacobs JF, et al. Prognostic significance and mechanism of Treg infiltration in human brain tumors. J Neuroimmunol. 2010;225(1-2):195-199. 
60. Gielen PR, et al. Increase in both CD14-positive and CD15-positive myeloid-derived suppressor cell subpopulations in the blood of patients with glioma but predominance of CD15-positive myeloid-derived suppressor cells in glioma tissue. J Neuropathol Exp Neurol. 2015;74(5):390-400.

61. Beauvillain C, et al. Neonatal and adult microglia cross-present exogenous antigens. Glia. 2008;56(1):69-77.

62. Müller S, et al. Single-cell profiling of human gliomas reveals macrophage ontogeny as a basis for regional differences in macrophage activation in the tumor microenvironment. Genome Biol. 2017;18(1):234

63. Müller S, et al. Peptide vaccine immunotherapy biomarkers and response patterns in pediatric gliomas. JCI Insight. 2018;3(7):98791.

64. Bronte V, et al. Recommendations for myeloid-derived suppressor cell nomenclature and characterization standards. Nat Commun. 2016;7:12150.

65. Yu N, et al. CD4(+)CD25 (+)CD127 (low/-) T cells: a more specific Treg population in human peripheral blood. Inflammation. 2012;35(6):1773-1780

66. Aktas E, Kucuksezer UC, Bilgic S, Erten G, Deniz G. Relationship between CD107a expression and cytotoxic activity. Cell Immunol. 2009;254(2):149-154.

67. Finck R, et al. Normalization of mass cytometry data with bead standards. Cytometry A. 2013;83(5):483-494.

68. Van Gassen S, et al. FlowSOM: Using self-organizing maps for visualization and interpretation of cytometry data. Cytometry A. 2015;87(7):636-645. 